\title{
Modeling the Interface Instability and Mixing Flow During the Process of Liquid Explosion Dissemination
}

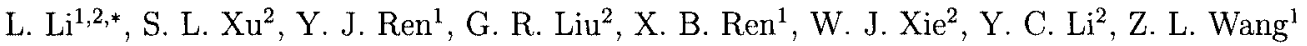 \\ ${ }^{1}$ Department of Engineering Mechanics, Tsinghua University, Beijing 100084, China \\ ${ }^{2}$ Department of Engineering Mechanics, University of Science and Technology of China, Hefei 230026, \\ China \\ Email: leirockyli@gmail.com or rockysys@public3.bta.net.cn
}

\begin{abstract}
The liquid flow during the process of liquid explosion dissemination is a typical complex high-speed unsteady motion with multi-scale in space and time. The motion of liquid flow may be partitioned to several stages. The first is initial liquid expansion by the action of shock wave and explosive gaseous products. The second is breakup of liquid annulus and turbulent mixing, which is called near-field flow. The third is two-phase mixing flow of gas and liquid drops, which is called far-field flow. To first stage, a compressible inviscid liquid model was used, while an elastic and plastic model was used to depict the expansion of solid shell. Numerical study in two dimensional has been made by using the Arbitrary Euler-Lagrange (ALE) methods. In near-field, the unstable flow of liquid annulus is dominated by many factors. (1) The shock action of gaseous expansive products. (2) The geometric structure of wave system in liquid. (3) The local bubble and cavitating flow in annulus, induce much of local unstable interface, tear up interfaces, and enhance the instability and breakup of liquid annulus. In this paper, some postulations are proposed that the cavitations in liquid annulus are induced by shock wave and the flow of liquid annulus is a two phase flow (liquid and a discrete bubble groups). Some experimental results will be presented that the breakup of interface and turbulent mixing is visualized qualitatively and measured quantitatively by using shadow photography method. The primary results are some flow patten of interfaces and some transient flow parameters by which the nonlinear character will be obtained, and provide an experiential support for modeling to unstable interface flow and turbulent mixing. The two-phase mixing flow between liquid drops and gas in far-field can be studied by numerical methods where the turbulent motion of gas phase is represented with $\mathrm{k}-\varepsilon$ model in Euler system, the motion of particle phase is represented with particle stochastic trajectory in Lagrange system, and the behavior of drops are calculated with breakup, coalescence, and evaporation models.
\end{abstract}

\section{REFERENCES}

1. Samirant M. Dynamic measurements in combustible and detonable aerosols. Propellants, Explosives, Pyrotechnics, 1989; 14: 47-56

2. Gardner DR. Near-field dispersal modeling for liquid fuel-air explosives. SAND90-0686, 1990

3. Ren Y J, Li L. A realization approach for the computation of elastic and plastic fluid flow. Chinese Reports, 2002.08

4. Ren X B, Li L, Li Y C. Numerical simulation of explosive dispersal of liquids in far-field period. Journal of Northeastern University (Natural Science), 27 S1: 190-193 